\title{
A Study of Pricing Decision for Open Source Software Value
}

\author{
Sangphil Kim ${ }^{1}$, Kook-Hyun Choi ${ }^{2}$, Jin-Young Park ${ }^{3}$ and Jong-Bae Kim ${ }^{4}$ \\ 1,3,4* Graduate School of Software, Soongsil University, Seoul, \\ 156-743, Korea \\ ${ }^{2}$ Department of IT Policy and Management, Graduate School of Soongsil \\ University, 156-743, Korea \\ ${ }^{1}$ sangphilkim@ssu.ac.kr, ${ }^{2}$ khchoi@tsline.co.kr, ${ }^{3}$ jin0902666@naver.com, \\ 4*kjb123@ssu.ac.kr
}

\begin{abstract}
Open Source Software, OSS, is about software that can freely use, copy, and release, source code using algorithm, function and product. People are not only expected to OSS will important roles in Cloud computing, big data, Internet of the Things and mobile era because unrestricted OSS's advantage, but also the increasing interest of OSS value valuation. However, OSS has a characteristic where everybody can release and share using source codes so it can't estimate. Now, there is no standard and/or mensuration about OSS value valuation. Therefore, this paper will estimate OSS values and compare commercial software price assessments with values on sale. Also, this adapted study methods suggest a result of software values compare analysis include suggest standard value valuation models for estimate software values. In this paper, it is expected that the suggested methods would help to various Open source way of value valuation.
\end{abstract}

Keywords: Open source software, OSS, Pricing decision, Value valuation

\section{Introduction}

\subsection{Study background}

Reasonable calculated software price movement has also played a key role as one of the major national development strategies in the 19th Administration. In May 2014, the Ministry of Science, ICT and Future Planning amended the Guidelines for Software Business Pricing to increase the commercial software maintenance rate from $8 \%$ to $12 \%$. The Ministry views the reason software firms receive only less than the reasonable prices are lax regulatory regulation. Under this assessment, the Ministry plans to have software ordering agencies preclude unfair behaviors proactively by timely applying software business-specific regulations [1].

OSS (Open Source Software) refers to the kind of software provided free of charge with open-source code execution program while allowing anyone to freely modify the source code and re-distribute. Anyone can read the source code and, if possible, correct all sorts of bugs or even remodel it to add a new function and participate in the software development. Given such a market-oriented development method, developers are rewarded with self-satisfaction or reputation, etc., instead of financial profits [2].

In this recognition, this study seeks to estimate OSS value and compare it with commercial software. To this end, based on the value assessment model proposed to evaluate software, SW values are compared and analyzed then the results are presented and tested in this study.

$4^{*}$ Corresponding author. Tel. : +82-10-9027-3148.

Email address: kjb123@ssu.ac.kr(Jong-Bae Kim). 


\subsection{Study Purpose}

This study presents an OSS value assessment model. In consideration of suppliers and demanders, the model estimates the currently utilized OSS values and analyzes if the shelved commercial software price is appropriate. To set the range of reasonable price, economic theories, business profitability, marketability and technological aspect were considered and the upper limit and lower limit of OSS price were determined. Price is suggested as in Figure 1. The upper price limit is purchase price including the utility value of the SW on the buyers' side and the financial amount they are willing to pay for such a utility value. The lower price limit is sales price including the total SW cost on the suppliers' side and expected profit.

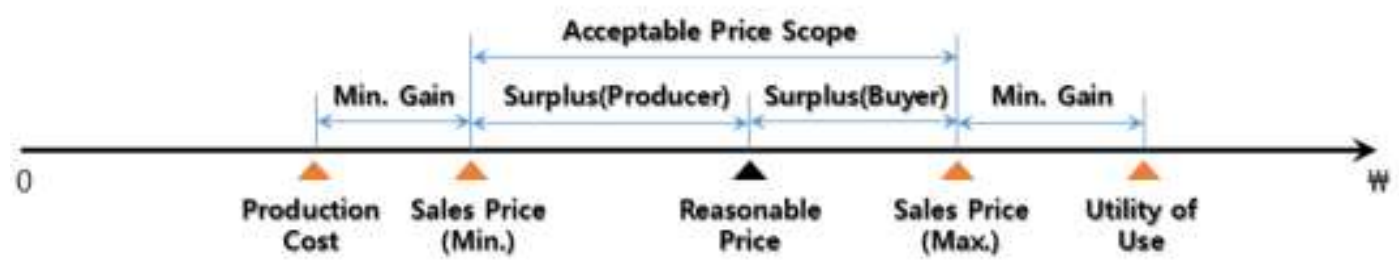

Figure 1. Acceptable Price Scope Estimation

That a certain SW is reasonably priced under the price reasonability analysis model means the specified price falls between the upper and lower price limits

\section{Related Study}

\subsection{Study on Software Value Assessment}

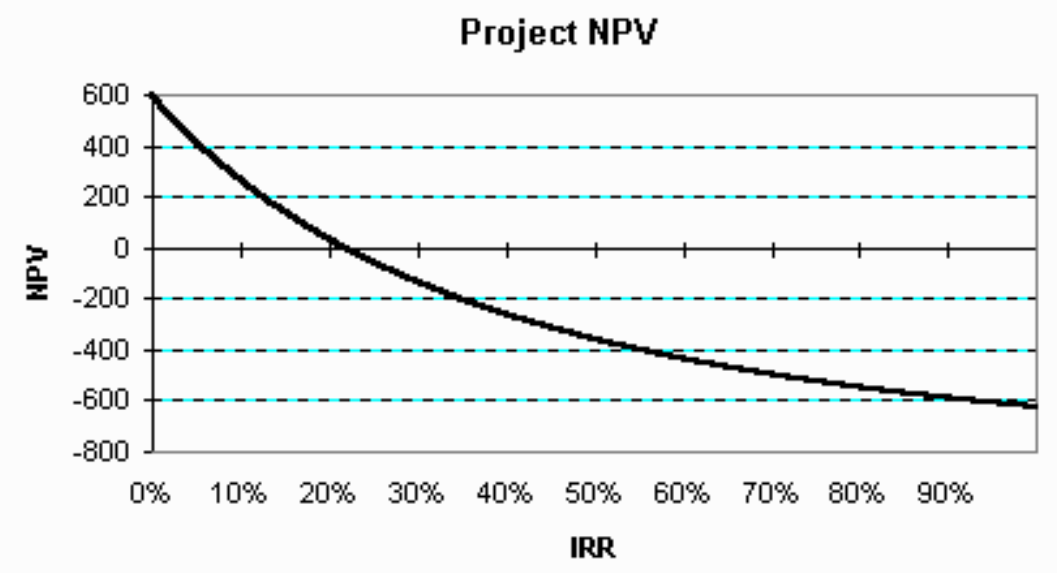

Figure 2. Calculate Net Present Value

There are multiple studies regarding SW as a product or service to apply price determination factors [3][4]. To analyze SW technologies, the basic data are collected and economic life cycle is estimated. Based on the previous SW-applied database, they estimate sales turnover in a statistical manner. If there is no target to assess, potential market size and market share are considered to estimate. Financial statements are analyzed to guess future cash flow and discount rate to produce business value in Figure 2. Lastly, SW technology contribution level is presumed to finalize SW technology value assessment. 
In the event of litigation due to SW copy or defect-related conflicts, the resulting damage amount was researched by a study to estimate corresponding compensation amount [5]. For damage compensation estimation, not only the SW technology values itself, but also involved SW production costs such as personnel expenses and manufacturing expenses are combined. In this aspect, there exist many common aspects with value assessment.

\subsubsection{Investment-Oriented Models}

Total Value of Opportunity (TVO) is a standard metrics-based approach invented by Gartner. Its aim is to judge the potential performance of a given IT investment over time. It centers on assessing risks and then quantifying the flexibility that a given option provides for dealing with each risk. Total cost of ownership is always used to characterize the overall cost of operation. Benefits are then judged using a broad range of organizational performance measures. The cost/benefit analysis must be comprehensive and appropriate to the situation, and it must describe the business case in terms that a nonIT executive can understand[6].

Because IT investment rarely produces immediate benefits, TVO also requires the business to quantify any probable future impacts of a given investment. This aspect is particularly attractive in the case of software assurance, because much of the investment in securing software is designed to ensure future advantage by preventing undesirable events. These benefits should be quantified based on assumptions that can be validated retrospectively or on data-based prospective estimates such as trend line analysis[7].

Any alteration in practice implies some form of substantive change, and organizational diagnostics essentially test an organization's ability to adapt to that change. The three types of risks (business, management, and technology) associated with change are assessed on five factors (Strategic Alignment, Risk, Direct Payback, Architecture and Business Process Impact). Those factors coincidentally happen to be Gartner's Five Pillars of Dynamic Benefits Realization.

The best practice in measurement simply requires the employment of a commonly accepted methodology to obtain the value estimates that underlie the Future Uncertainty factor. The aim of the measurement process is to enable a conventional business analysis that is capable of communicating the value proposition to a general audience. The key to this part of the approach is a small set of agreed-upon business metrics. The use of common metrics ensures understanding between major stakeholders. Consequently, the development of those metrics is critical to the process.

Like TVO, Total Economic Impact (TEI) is meant to integrate risk and flexibility into a model that will support intelligent decisions about IT investment. TEI is a proprietary methodology of the Giga Group that allows an organization to factor intangible benefits into the equation by assessing three key areas of organizational functioning[8].

Flexibility is a function of the value of the options the investment might provide. It can be described in terms of enhanced financial value or increased communication potential or on the basis of potential future increases in business value. The supporting methodology can describe the actual value of the options that are available at the decision point, or it can describe the value of an option to be exercised later (for instance, an assumption that the future market share will increase as a result of an increase in assurance).

The cost analysis takes a TCO-like approach in that it considers ongoing operating costs along with any initial capital outlay. It factors both IT budget expenditures and the allocated cost of the overall organization control structure into the assessment.

Benefits are expressed strictly in terms of increased business value. That expression includes any value that can be identified within the IT function as well as any value that is generated outside of IT. Thus, benefit assessments also look at the project's business 
value and strategic contribution and consider how appropriately the investment aligns with business unit goals.

TEI is one of the softer kinds of value estimation methodologies and seems to be most useful when an organization's aim is to align a technology investment with a business goal or to communicate the overall value proposition of an initiative. TEI's primary purpose is to underwrite sound business decisions, given a set of alternatives. It does that by communicating each alternative's full value in business terms. Thus, TEI can be used to justify and relate a proposed direction to any other possible directions. That creates a portfolio view of the entire IT function, which enables good strategic management practice. Since understanding the overall impacts is obviously one of the primary goals of any software assurance valuation process, TEI is an attractive approach.

In order for it to be acceptable, the cost of the software assurance process has to be justifiable in hard economic terms. But more important, that estimated cost must be available when needed. The problem is that most valuation techniques require long periods of data collection in order to produce valid results. The aim of Microsoft's Rapid Economic Justification (REJ) is to provide a quick and pragmatic look at the value of the investment, without taking the usual lengthy period of time to collect all the necessary operational data. Like the Total Economic Impact approach, REJ seeks to flesh out traditional TCO perspectives by aligning IT expenditures with business priorities [9].

\subsubsection{Cost-Oriented Models}

Economic Value Added (EVA) approaches IT investment as a value proposition rather than as a cost. That is, EVA attempts to describe all the ways a prospective investment might leverage organizational effectiveness. EVA approaches this question by looking at a function in terms of the cost saving it might create when compared to the cost of obtaining the same function through external providers at a market rate. Once the comparative market value is determined, EVA quantifies the difference between the market price and the actual cost of providing the prospective function. That difference is the net operating benefit [10].

Costs are characterized by such things as capital outlay and opportunity cost. The aim of an EVA comparison is to determine whether the market value of any investment, after the actual costs are deducted, is positive. Therefore, EVA requires a careful accounting of all expenditures as well as an honest estimate of any opportunity cost [11].

An EVA analysis demands that everything from initial cash outlays to maintenance and training - including any expenditure that is legitimately part of the initiative-is charged against profit. EVA is then calculated as the Net Operating Profit After Tax (NOPAT) minus the Weighted Average Cost of Capital (C) as adjusted by a range of proprietary adjustments (K) that are provided as a service by Stern \& Stewart [11].

Those adjustments include such things as the "amortization of goodwill or capitalization of brand advertising." The advantage of EVA is that it produces a single financial index that can be used to characterize a diverse set of potentially contradictory directions. Approached as a tradeoff between total investment cost and potential value, EVA is a good way to gauge the impact of any process such as assurance on overall profitability. Beyond the general cost/benefit view however, EVA is really only useful when it leads into the use of another more precise valuation methodology [12].

Economic Value Sourced (EVS) sets out to quantify the value gained for every dollar invested. The investment in software assurance is always speculative because the risk and reward structure is hard to quantify. For instance, how do you assign a quantitative value to the increased customer trust that a secure software assurance function provides? In response to questions like that, EVS extends the analysis beyond the EVA approach by factoring risk and time considerations into the equation.

EVS assumes that IT investment decisions can be valued based on three strategic factors: reduction of risk, increase in productivity, and decrease in cycle time. Traditional 
return on investment (ROI) measures such as risk reduction savings or marginal productivity increases are the typical basis for quantifying value.

In addition, EVS adds standard timing factors such as flexibility. For instance, EVS asks such questions as "If the investment represents continuing cost, and how quickly can those costs be adjusted to decreases in profitability". Finally, risk-based considerations, such as the overall impact of the proposed investment on performance, interoperability, resiliency, or security of the operation are also factored in.

EVS is an attractive approach because it allows for considerations outside of the traditional economic rate of return - considerations through which many of the indirect, abstract, or qualitative economic benefits of investment in software assurance can be understood and justified.

Total Cost of Ownership (TCO) is one of the older, and more traditional, cost-based valuation approaches. It assesses an investment based strictly on its total direct and indirect costs. TCO aligns those costs with ongoing business performance in order to evaluate total value but does not assess risk or provide a means to ensure alignment with business goals [12].

When incorporated with a classic financial analysis such as ROI, TCO can provide a true economic value for any given investment. TCO takes a holistic view of total organizational cost over time. Ideally, it will let the manager calculate a projected rate of return on any investment based on the initial capital outlay, as well as all the aspects of the continuing cost of operation and maintenance. That cost estimate typically includes such ancillary considerations as physical space, security and disaster preparedness, training, and ongoing support. That's why TCO is sometimes referred to as Total Cost of Operation.

Benefit is generally calculated using an estimate of the cost that would accrue if a function or service were absent. For instance, TCO asks what the cost to the organization would be if a system failed or experienced a security incident. It then treats that cost value as a risk avoidance benefit. By treating incident cost that way, TCO provides a good running benchmark of the financial value of an overall risk mitigation program for software assurance.

TCO can be used to monitor the overall effectiveness of any assurance program by comparing the running cost of maintaining a given level of security to existing financial data about the cost of the incidents the program is designed to prevent. For instance, if a given level of assurance is established to prevent buffer-overflow attacks, the national average cost of those attacks can be used as an index of the benefit that would be gained by preventing them.

Because it is strictly cost centered, TCO is best used for cost rather than value estimation. However, TCO also works well in conjunction with methodologies such as the Balanced Scorecard to provide an easy to understand picture of the cost side of the proposition.

\subsubsection{Quantitative Estimation Models}

Real Options Valuation (ROV) aims to put a quantitative value on operational flexibility. It allows an organization to value any investment that will underwrite or create a more relevant and responsive operation. Thus, ROV can be used to value technological investment.

ROV centers on ensuring maximum flexibility in the deployment of technological assets. Using this approach, an organization can determine the value of an investment by focusing on the likely consequences of a particular action over time [13].

In most instances, those outcomes are characterized by assumptions about future performance. However, no set of assumptions is going to provide a perfect forecast. The best approach to the ROV process is to derive a value for every feasible option [13]. 
As a consequence, much of ROV involves identifying every factor that might be involved in or impacted by a given decision and then estimating the likelihood of occurrence. Thus, ROV is based on decision variables, stochastic assumptions and deterministic assumptions.

Real options have concrete outcomes. Thus the decision rules for a exercising a real option must be referenced to observable behaviors that can be used to assess the performance of every variable associated with it. These behaviors must be observable and documented for a given period prior to the point where the decision is made. For example, a decision to add an assurance practice might be based on the known occurrences and costs of the threats that practice was meant to address over the past year of operation.

The problem with ROV is that it is, by necessity, complex, so it works best in situations that are well defined or where experience exists. Thus, ROV models are effective in estimating the likelihood of stock options or pork bellies. However, since the process of assurance is not yet well understood, the construction of the finite model for it is, at best, an exploratory effort [14].

Applied Information Economics (AIE) is perhaps the most rigorously quantitative methodology in this set. It centers on the use of probabilistic models to reduce uncertainty. It is assumed that if the appropriate amount of data can be collected, it is possible to calculate the fiscal value of any option [15].

Since all decisions involving deployment of the software assurance function involve the estimation of probabilities of both benefit and failure, it is hypothetically possible to build a sufficiently accurate picture of the financial risks and returns of any given decision option, or a related set of options, using AIE. This will allow the decision maker to understand the exact probabilities of success. This knowledge can then theoretically allow decision makers to balance their assets and activities in such a way that they will exhibit the best risk-reward characteristics [15].

The analysis process itself involves classic actuarial estimation. Actuarial statistics are used in order to quantify the consequences of a given decision, which provides a proper understanding of risk and return.

Applied Information Economics computes the value of additional information. The aim is to model uncertainty quantitatively and then compute the value of marginal uncertainty reductions. The AIE process is based on Hubbard's Clarify, Measure, Optimize approach, which aims to isolate and clarify the precise set of variables that are involved in and affect the decision. Such isolation and clarification allows AIE to provide specific information for decision makers.

For example, most decisions about software assurance are made based on the probability of harm. Thus, a manager might estimate that a given program would have a likelihood of $20 \%$ of failing or being exploited. AIE would restate that estimate in terms of the probabilities that a certain type of virus would be able to exploit that code, versus the likelihood that it could be compromised by a range of other attack types. This sort of detail makes it easier to estimate the long-term value of the decision to increase or decrease the assurance activity[15].

AIE analysis is considered by its proponents to be the only truly scientific and theoretically based methodology available. Its ideal outcome is an actuarial risk-versusreturn statement about the probabilities of the success of a given decision. In order to do that, AIE integrates classic principles of economics, actuarial science, and decision theory into a single approach that theoretically supports proper decision making about how to conduct business operations.

\subsection{South Korean OSS Value Assessment Situation}

The South Korean government announces its favorable position to OSS issues. It has formed Open Software Activation Forum to search for how to facilitate OSS development 
and application. However, related specific project or legislation has yet to be specifically shaped [16-17].

The country has not prescribed OSS value assessment scheme while only having laws to support the necessary assessment procedures for technology transfer or security provision for financial support. Under the Act on Special Measures for The Promotion of Venture Businesses and Technology Transfer and Commercialization Promotion Act, the Korea Institute of Science and Technology Information (KISTI) was designated as a technology assessment organization. In 2004, the online value assessment system was developed, offering the SW value assessment model as in the equation (1) below;

$$
\mathrm{V}=\sum_{t=1}^{T} \frac{F C F_{t}}{(1+r)^{t}} \times T \cdot F .
$$

The final technology value assessment amount $(\mathrm{V})$ income approach is used to multiply the expected Free Cash Flow (FCF) during the expected economic lifespan of the target technology by Technology Factor (T.F.). FCF is the amount of adding depreciation cost to post-tax operating profit less capital outlays and net working capital changes.

\section{Oss Value Estimation Model}

\subsection{Value Assessment Approach}

\subsubsection{Income Approach Value Assessment (Profitability)}

The income approach is to estimate future expected earnings from a targeted asset and calculate its current value [17-18]. The current value of the corresponding asset's economic gain throughout its lifespan is assessed. Regardless of intellectual property right creation or construction cost, the approach focuses on the profit generation ability [19].

If the data set is not enough to calculate replacement cost, this approach could be a practical assessment method to calculate current value by using future value [20]. However, it is difficult to precisely anticipate the cash flow size and time point, intellectual property assets' practical application range and level.

\subsubsection{Market Approach Value Assessment}

The market approach is to comprehensively compare the market transaction information of assets similar to the targeted one to guess the target IPR value [17]. In other words, the basic principle of market approach is to combine the current value of asset with the market-determined asset value to estimate the current value of future earnings. The market approach functions more effectively when there are multiple suppliers and buyers.

General manufacturing products have a clear set of assessment criteria. Thus, their relative quality evaluation is easier and suppliers open product prices for customers to decide. But software is different as the more expensive, the more functions they tend to have or the faster they are, for instance. Unlike the manufactured goods, SW assessment criteria are unclear and though there is SW development cost, there is no manufacturing cost, making it difficult to determine a reasonable price.

\subsubsection{Cost Approach Value Assessment (Technological)}

The cost approach is to calculate the financial amount of resources necessary to produce the equal value to the target asset's value and estimate its future earnings. For value estimation, the approach requires to calculate CRN (Cost of Reproduction New) necessary to gain the same thing as the target asset or Cost of Replacement necessary to obtain an asset with the equal effectiveness to the target asset [19]. 
Assessment result is accurate if there is large market data accumulation. But as the method does not base on profitability, expected profit is hardly considered. Social structure or market characteristics are hardly reflected.

\subsection{Composition of OSS Price Determination Model}

The OSS price determination model combines the three different approaches to present price upper limit and lower limit. The model estimates a reasonable price by employing the income approach and cost approach to process the future value estimation and product production/development cost and by employing the market approach to evaluate product market value and produce the price upper and lower limits.

\subsection{Second-order headings}

The reasonable price $\mathrm{NV}_{\mathrm{j}}$ can be measured through income approach method and via the cost spent on software production. This study suggests Equation (2) as the method to calculate a reasonable price, which is a mixed form of income approach method and cost approach method.

$$
N V_{j}=\frac{\frac{1}{n} \sum_{i=1}^{n} I C A+\left(1-\frac{1}{n} \sum_{i=1}^{n} E X P A\right)}{2}
$$

$\mathrm{NV}_{\mathrm{j}}$ : The reasonable price for OSS category $\mathrm{j}$

ICA : Cash flow and technology needs cost during economic life cycle via income approach method

EXPA : The cost spent on software development via cost approach method

The classification via category $\mathrm{j}$ was done by the purpose of the software. For example, there are differences between ICA and EXPA for server software and embedded software as shown in [Table 1], but we applied weighted values by categories in order to decrease error range. It is to use $\mathrm{NV}_{\mathrm{j}}$ value which includes the weighted value for the category when calculating price range by using market approach method.

Table 1. Classification via Software Category Usage

\begin{tabular}{|c|c|}
\hline \multicolumn{2}{|c|}{ Software category } \\
\hline For desktop use & For business use \\
\hline For server use & For embedded use \\
\hline For Cloud and Big data use & For quality verification use \\
\hline
\end{tabular}

In order to calculate a price range, we suggest the upper limit and lower limit for software price using applied $\mathrm{NV}_{\mathrm{j}}$ value as shown in Equation (3) and (4).

$$
\begin{gathered}
\mathrm{HNV}=N V_{j}+\frac{1}{n} \sum_{i=1}^{n} M K A \\
\mathrm{LNV}=N V_{j}-\frac{1}{n} \sum_{i=1}^{n} M K A
\end{gathered}
$$

HNV : Upper limit for OSS reasonable price

LNV : Lower limit for OSS reasonable price

MKA : Market variable considering rarity and approachability of technology

via market approach method 
We calculate the upper and the lower limits for the OSS reasonable price by applying market variable MKA to $\mathrm{NV}_{\mathrm{j}}$. The market variable is a value where rarity and approachability within the market and the price range considering market conditions can be set by applying it onto the reasonable price.

\section{Conclusion}

The study established a new model by combining the presently-utilized models based on value assessment and suggested a new assessment model for OSS value evaluation. We calculated the reasonable price for OSS by using a mixed method of income approach method and cost approach method along with the market variable considering the market conditions through market approach method. However, the model suggested by this study is not practical enough to be used for direct evaluation as there are more researches required on setting weighted values and measuring market variables upon category settings for reasonable prices. In this aspect, future studies will need to select assessment targets and apply and test the proposed model. The model needs to be utilized to estimate price and compared with existing commercial software for verification. This study is expected to ultimately serve as the basis for OSS activation and software assessment model.

\section{References}

[1] S. Kim, K. H. Choi, J. Y. Park, C. H. Woo and J. B. Kim, "Software Pricing Decision Model: Based on Open Source Value Valuation”, ICFICE 2016, vol. 8, no. 1, (2016).

[2] T. F. Smith and M. S. Waterman, "Identification of Common Molecular Subsequences", J. Mol. Biol., vol. 147, (1981), pp. 195-197.

[3] P. May, H. C. Ehrlich and T. Steinke, "ZIB Structure Prediction Pipeline: Composing a Complex Biological Workflow through Web Services", In: Nagel, W.E., Walter, W.V., Lehner, W. (eds.) EuroPar 2006. LNCS, Springer, Heidelberg, vol. 4128, (2006), pp. 1148-1158.

[4] I. Foster and C. Kesselman, "The Grid: Blueprint for a New Computing Infrastructure", Morgan Kaufmann, San Francisco, (1999).

[5] K. Czajkowski, S. Fitzgerald, I. Foster and C. Kesselman, "Grid Information Services for Distributed Resource Sharing", In: 10th IEEE International Symposium on High Performance Distributed Computing, IEEE Press, New York, (2001), pp. 181-184.

[6] A. Audrey, "The Total Value of Opportunity Approach", CIO, (2003).

[7] M. M. Adam, K. Rajiv and D. Sarv, "Measuring Business Value of Information Technology in EBusiness Environments", Journal of Management Information Systems, vol. 21, (2004), pp. 11-16.

[8] R. Wang and J. Erickson, "The Financial Impact of Packaged Applications, a Tool for Comparing the ROI of Enterprise Applications", Cambridge, MA: Forrester Research, Inc., (2006).

[9] MSDN, "The Microsoft Security Development Lifecycle (SDL)", Measurable Improvements for Flagship Microsoft Products, (2008).

[10] P. Justin, D. John, P. Karl and P. Jorge, "EVA and Corporate Portfolio Strategy", Evaluation, vol. 3, no. 9, (2001).

[11] M. Ben, "All About EVA", Investopedia.com, (2003).

[12] M. Tracy, “A Buyer's Guide to IT Value Methodologies”, CIO Magazine, (2002).

[13] A. L. Timothy, "Investment Opportunities as Real Options: Getting Started on the Numbers", Harvard Business Review, (1998).

[14] N. James, "Hybrid Real Options Valuation of Risky Product Development Projects", Cleveland, OH: Booz-Allen \& Hamilton, (2001).

[15] H. Douglas, "Everything is Measurable", CIO Enterprise Magazine, (2008).

[16] I. Foster, C. Kesselman, J. Nick and S. Tuecke, "The Physiology of the Grid: an Open Grid Services Architecture for Distributed Systems Integration", Technical report, Global Grid Forum, (2002).

[17] I. S. Hwang, "A Study on the Business Value of Products Considering Cross Selling Effect", Asia Pacific Journal of Information Systems, vol. 15, no. 3, (2005).

[18] K. Schmid and M. Verlage, "The economic impact of product line adoption and evolution", IEEE Software, vol. 19, no. 4, (2002), pp. 50-57.

[19] I. Bardhan, S. Bagchi and R. Sougstad, "Prioritizing a portfolio of information technology investment projects", Journal of Management Information Systems, vol. 21, no. 2, (2004), pp. 33-60.

[20] Z. Irani, "Information Systems Evaluation - navigating through the problem domain", Information and Management, vol. 40, (2002), pp. 11-24. 


\section{Authors}
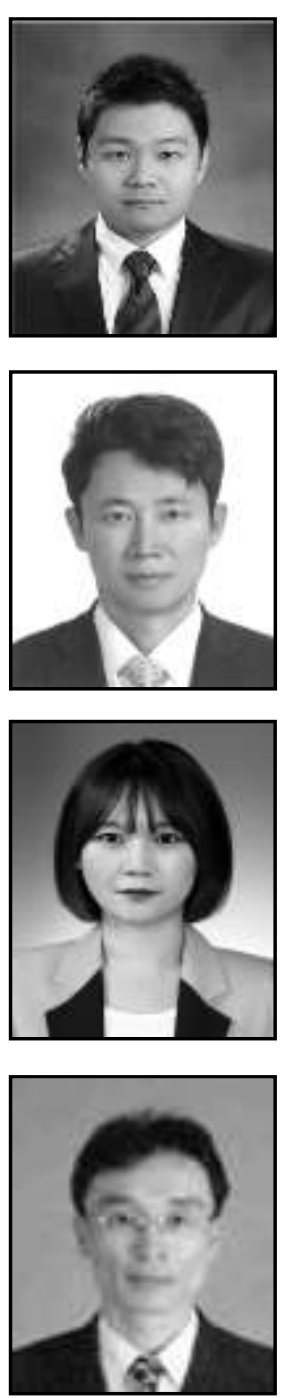

Jong-Bae Kim, he received his bachelor's degree of Business Administration in University of Seoul (1995) and master's degree (2002), doctor's degree of Computer Science in Soongsil University, Seoul (2006). Now he is a professor in the Graduate School of Software, Soongsil University, Korea. His research interests focus on Software Engineering, and Open Source Software. 